\title{
Effects of silica-rich water on systemic and peritoneal inflammation in rats exposed to chronic low-level $(900-\mathrm{MHz})$ microwave radiation
}

\author{
Boris Djindjic ${ }^{1}$, Tanja Dzopalic ${ }^{2}$, Momir Dunjic ${ }^{3}$, Dejan Krstic ${ }^{4}$, Zoran Radovanovic ${ }^{5}$, \\ Jelena Milenkovic ${ }^{1}$, Natasa Djindjic ${ }^{2}$ and Biljana Bozic-Nedeljkovic ${ }^{6}$ \\ ${ }^{1}$ Department of Pathophysiology, Faculty of Medicine, University of Nǐ̌, Niš, Serbia \\ 2 The Scientific Research Center for Biomedicine, Faculty of Medicine, University of Niš, Niš, Serbia \\ ${ }^{3}$ Faculty of Pharmacy, The University Business Academy, Novi Sad, Serbia \\ ${ }^{4}$ Faculty of Occupational Safety, University of Niš, Niš, Serbia \\ ${ }^{5}$ Institute of Radiology, Clinical Center Nis, Faculty of Medicine, University of Niš, Niš, Serbia \\ ${ }^{6}$ Faculty of Biology, University of Belgrade, Belgrade, Serbia
}

\begin{abstract}
Immunomodulating effect of silica-rich water represents a novel field for research, especially regarding its features toward environmental pollutants. The aim of our study was to evaluate the effects of silica-rich water intake on systemic and peritoneal inflammation in rats that were chronically exposed to the low-level microwave (MW) radiation from mobile phones. Wistar Albino rats were exposed to $900 \mathrm{MHz} \mathrm{MW}$ radiation for 3 months. The four-treatment model involved rats with standard water (SW) or experimental silica-rich water intake (EW). Peritoneal macrophages (PMs) were harvested using peritoneal lavage and divided into nonstimulated and lipopolysaccharide (LPS) stimulated subgroups. The MW-exposed rats with silicarich water (MW+EW) had lower serum tumor necrosis factor $\alpha$ (TNF- $\alpha$ ) and interleukin 2 (IL-2) levels, but higher IL-10 levels, than MW+SW rats $(p<0.05)$. The higher TNF- $\alpha$ production by non-stimulated MW exposed PMs was ameliorated by the silica-rich water $(p<0.01)$. The MW exposition suppressed LPS potential for TNF- $\alpha$ synthesis in both water type groups, with greater suppression in animals that took standard water. Our results show the modulating effect of silicarich water toward $\mathrm{MW}$-induced systemic and peritoneal inflammation, which reflects the water ability to shape monocyte plasticity, thereby altering the balance between their proinflammatory and anti-inflammatory properties.
\end{abstract}

Key words: Silicon dioxide - Tumor necrosis factor $\alpha$ - Interleukin 10 - Peritoneal macrophages - Adhesion molecules

\begin{abstract}
Abbreviations: EW, experimental silica-rich water; GSM, global system for mobile communication; IL, interleukin; LPS, lipopolysaccharide; MW, microwave; PBS/NaEDTA, phosphate-buffered saline/ tetrasodium-ethylenediaminetetraacetate; PMs, peritoneal macrophages; SAR, specific energy absorption; SD, standard deviation; sICAM-1, soluble intercellular adhesion molecule $1 ; \mathrm{SiO}_{2}$, silicon dioxide; sVCAM-1, soluble vascular cell adhesion molecule 1; SW, standard water; TNF- $\alpha$, tumor necrosis factor $\alpha$.
\end{abstract}

Correspondence to: Jelena Milenkovic, Department of Pathophysiology, Faculty of Medicine University of Niš, Blvd. Zorana Djindjica 81, 18000 Niš, Serbia

E-mail: jelenaradovic982@gmail.com 


\section{Introduction}

Mobile phones use and wireless communication are considered inseparable part of modern way of life. Rapidly increasing use of mobile phones is believed responsible for health effects of non-thermal microwave (MW) exposures (Baan et al.2011). Discrete physiological alterations induced by the chronic exposition to the low levels of MW radiation launched investigations aiming for identification of sensitive biomarkers of these processes. Current evidence indicates possible alterations in number and activity of immunocompetent cells under MW exposure, making these changes suitable for novel biomarkers research. However, the results are still inconsistent (Szmigielski 2013). Silica or silicon dioxide $\left(\mathrm{SiO}_{2}\right)$ is one of the most common mineral in earth's soil and is found in drinking water as water-soluble forms or silicic acid. Water soluble silicates are important sources of bioavailable silicon forms (Robberecht et al. 2009). The mixture of sodium silicate causes non-specific immuno-stimulating effects and increased phagocytic activity in animals upon releasing ortho-silicic acid (Koo et al. 2006). The similar mechanism is proposed for certain types of zeolites such as zeolite A and the natural zeolite (Munjas Jurkić et al. 2013). The immunomodulating effects of silica-rich waters, in the presence of chronic exposition to MW radiation, are an interesting concept for research, especially regarding its protective features. Studies of MW radiation effects on peritoneal macrophages (PMs) are scarce, although they represent important regulators and markers of gastrointestinal epithelium integrity and inflammation. Accumulating data emphasize the important role of intestinal barrier for various diseases pathogenesis, especially autoimmune diseases. Activation of the immune system in MW environment is supposed to take part in autoimmune and proinflammatory disorders (Stankiewicz et al. 2006; Cassado et al. 2015). The aim of our study was to evaluate the effects of silica-rich water intake on systemic and peritoneal inflammation in rats that were chronically exposed to low level $(900 \mathrm{MHz})$ MW radiation from mobile phones.

\section{Material and Methods}

\section{Animals}

One-month-old male Wistar Albino rats $(n=28)$ were bred at the Vivarium of the Scientific Research Center for Biomedicine, Faculty of Medicine University of Nis, Serbia, under conventional laboratory conditions. The rats were handled in accordance with EU guidelines for the accommodation and care of animals used for experimental and other scientific purposes (2010/63/EU). Before initiating the experiment, the rats were housed collectively in standard laboratory cages (seven animals in each $90 \times 120 \times 30 \mathrm{~cm}(\mathrm{~W} \times \mathrm{L} \times \mathrm{H})$ cage) for 2 weeks. The housing room was maintained at $24^{\circ} \mathrm{C}$ with $42 \pm 5 \%$ relative humidity and had a $12-12 \mathrm{~h}$ light-dark cycle (light on between 06:00 and 18:00 h). Standard laboratory chow was the same for all animals. Food and water were available ad libitum. All animal experiments were approved by the Animal Ethics Board of the Faculty of Medicine in Nis (No: 323-07-06862/2016-05/7) and were performed according to board guidelines. The rats' general health was monitored every day.

\section{Experimental design}

Chronic exposure to low level of MW from mobile phone model with four treatment arms was employed. The MW exposed groups were exposed to a non-thermal level of 900-MHz (GSM - Global System for Mobile communication) continuous MW, 4 hours per day during 3 months. The MW radiation was produced by a GSM emission module with stick antenna connected to a Communication Test Set with PC and appropriate software module. The emission module and exposure time were automatically controlled by a computer. The animals were kept in a pure (i.e. lacking any metallic fittings) polycarbonate cages. Emission antenna was fitted in perforated polycarbonate box situated in the middle of the cage's roof to ensure uniform distance from the corners. The intensity of the electromagnetic field components was measured several times in four points and kept constant during experiment. Electrical field was estimated by electromagnetic field meter Aaronia Spectran HF6080 with mean $\mathrm{E}=0.88-1.9 \mathrm{~V} / \mathrm{m}$ and mean magnetic field $\mathrm{B}=2.3-4.9 \mathrm{mT}$ according to distance from antenna. The whole-body specific energy absorption (SAR) rate was estimated as $0.048-0.142 \mathrm{~W} / \mathrm{kg}$ using data for a rotating ellipsoid model of a rat. The temperature inside polycarbonate box is monitored by a field thermal detector. The temperature rise was less than $0.1^{\circ} \mathrm{C}$ during the exposure. The housing room was without near sources of electromagnetic field. One by one, cages were moved to the exposure room to be exposed or sham exposed for $4 \mathrm{~h}$ /day. The everyday shifting of groups was applied to avoid exposure or sham exposure at the same time. The 28 male study rats were divided into four groups of seven animals. The control rats only received standard tap water (SW group; $6 \mathrm{mg} / \mathrm{l}$ of silicon determined by ICPOES (iCAP 6500 Duo, Thermo Scientific, United Kingdom) or experimental (silica-rich) water (EW group; $19 \mathrm{mg} / \mathrm{l}$ of silicon) ad libitum. The experimental water was made by adding a solution of sodium-silicate (sodium silicate, $\mathrm{Si}$ $1.00 \mathrm{~g} / \mathrm{l}$, Merck, Germany) in standard tap water. Average concentration of silica in natural water ranges between 1.0 and $20.0 \mathrm{ml} / \mathrm{l}$, thus we examined the effects of waters containing high $(19 \mathrm{mg} / \mathrm{l})$ or low $(6 \mathrm{mg} / \mathrm{l})$ silica levels. The $\mathrm{MW}+\mathrm{SW}$ and $\mathrm{MW}+\mathrm{EW}$ groups were chronically exposed 
to the low level of MW radiation and received the standard and experimental water supply, respectively. After 3 months, the animals were anesthetized by intraperitoneally injecting ketamine $\mathrm{HCl}(50 \mathrm{mg} / \mathrm{kg}$ ) and then were sacrificed. During the study, none of the animals were subjected to procedures that caused pain or discomfort.

\section{Isolation of peritoneal cells}

Peritoneal cells were harvested from the 28 rats by peritoneal lavage with $20 \mathrm{ml}$ of ice cold phosphate-buffered saline/ tetrasodium-ethylenediaminetetraacetate (PBS/NaEDTA). The harvested cells were then purified by using 40\% OptiPrep gradient. The purity of the peritoneal macrophage population obtained by this method was $85 \%$, as determined by staining with FITC-conjugated anti-CD68 antibodies (AbD Serotec, Oxford, UK). The purified cells were then seeded in 96-well plates $\left(10^{5}\right.$ cells per well) and cultured in standard conditions $\left(5 \% \mathrm{CO}_{2}, 37^{\circ} \mathrm{C}\right)$. In parallel, the purified cells were stimulated with lipopolysaccharide (LPS) $(10 \mu \mathrm{g} / \mathrm{ml})$. The cytokines release was measured after $24 \mathrm{~h}$ and phagocytic activity after 72 h cultivation in vitro.

\section{Detection of cytokines in the serum and cell culture medium}

Blood samples were drawn by cardiac puncture from the animals in terminal anesthesia and were placed in vials with EDTA. The serum was separated and subjected to ELISA. The following serum cytokines were measured: tumor necrosis factor $\alpha$ (TNF- $\alpha$ ), interleukins IL-2 and IL-10, soluble intercellular adhesion molecule 1 (sICAM-1), and soluble vascular cell adhesion molecule 1 (sVCAM-1). All ELISAs were performed following the instructions of the manufacturer (R\&D Systems, Minneapolis, USA). The sensitivity of the TNF- $\alpha$ (Cat. No. RTA00), IL-2 (Cat. No. R2000), IL-10 (Cat. No. R1000), sICAM-1/CD54 (Cat. No. RIC100), and sVCAM-1/CD106 (Cat. No. DVC00) ELISAs was up to $5 \mathrm{pg} / \mathrm{ml}$, up to $15 \mathrm{pg} / \mathrm{ml}$, up to $10 \mathrm{pg} / \mathrm{ml}, 1.2-4.1 \mathrm{pg} / \mathrm{ml}$ (assay range $=31.2-2.000 \mathrm{pg} / \mathrm{ml}$ ), and $0.17-1.26 \mathrm{ng} / \mathrm{ml}$ (assay range $=6.3-200 \mathrm{ng} / \mathrm{ml}$ ), respectively. The amount of TNF- $\alpha$, IL-10, and sICAM-1 that was released by the non-stimulated or LPS-stimulated PMs was determined by subjecting the cell supernatants after $24 \mathrm{~h}$ cultivation in vitro, to the same ELISAs described above.

\section{Phagocytosis assay}

The phagocytic ability of the cultured PMs was assessed by measuring Neutral Red uptake using the technique described by (Repetto et al. 2008). The PMs were placed in a 96-well plate and cultured at $37^{\circ} \mathrm{C}, 5 \% \mathrm{CO}_{2}$ for $72 \mathrm{~h}$, after which $50 \mu \mathrm{l}$ of Neutral Red was added to each well (dilution $=1: 300$ ). After incubating the plates for $4 \mathrm{~h}$, the supernatants were dis- carded, and the cells were washed three times with PBS ( $\mathrm{pH}$ 7.2-7.4). The washed macrophages were then resuspended in $100 \mu \mathrm{l} /$ well of cell lysis solution (ethanol and $1 \%$ acetic acid at a ratio of 1:1) and cultured for $2 \mathrm{~h}$. The phagocytic activity of the cells was determined by measuring the absorbance at $540 / 650 \mathrm{~nm}$ by using an ELISA reader. All determinations were conducted in quadruplicate.

\section{Statistical analysis}

All data were presented as means \pm standard deviation (SD). The animal groups were compared in terms of 90-day body weights, serum cytokine levels, and PMs cytokine production and phagocytosis by using the Student's $t$-test. $p$ values $<0.05$ were considered to indicate statistical significance. The data were analyzed by using a commercially available statistics software package (SPSS $16.0^{\circ}$ for Windows, Chicago, USA).

\section{Results}

The four animal groups did not differ significantly in terms of the average body weights at the end of the 90-day study period (Table 1).

\section{The effects of chronic MW exposure and silica intake on serum cytokine levels}

The chronic MW exposure exerted a dual effect on the serum TNF- $\alpha$ levels depending on the water type used (Figure 1A). The MW+EW rats had slightly lower TNF-a levels than sham-exposed EW controls, while MW+SW rats had significantly higher TNF- $\alpha$ levels than their SW controls $(p<0.05)$. The MW+EW rats had significantly lower TNF- $\alpha$ levels than the MW+SW $(6.7 \pm 2.1 v s .12 .0 \pm 3.5 \mathrm{pg} / \mathrm{ml} ; p<0.01)$. Taken together, TNF- $\alpha$ was increased in the rats exposed to chronic

Table 1. Average body weight (g) of the rats at the end of the 90-day study period

\begin{tabular}{lccccc}
\hline Group & Mean & SD & SE & 95\% CI for mean & $n$ \\
\hline EW & 303.7 & 30.2 & 10.6 & $278.5-329.0$ & 7 \\
MW+EW & 327.5 & 31.9 & 11.2 & $300.7-354.2$ & 7 \\
SW & 312.5 & 36.9 & 13.0 & $281.6-343.3$ & 7 \\
MW+SW & 311.2 & 38.7 & 13.6 & $278.9-343.6$ & 7 \\
\hline
\end{tabular}

$\mathrm{EW}$, sham-exposed rats with silica-rich water supply; MW+EW, MW exposed with silica-rich water supply; SW, sham-exposed rats with standard water supply; MW+SW, MW exposed with standard water supply; SD, standard deviation; SE, standard error; $\mathrm{CI}$, confidence intervals. Student's $t$-test shows the four groups did not differ in terms of body weight. 
MW radiation, but silica-rich water intake seemed to maintain its systemic levels. The MW+SW rats had significantly higher IL-2 serum levels than SW controls, and MW+EW group $(p<0.05)$. This effect was not observed in MW+EW group when compared to EW controls. The silica-rich water suppressed the ability of chronic MW exposure to increase

A

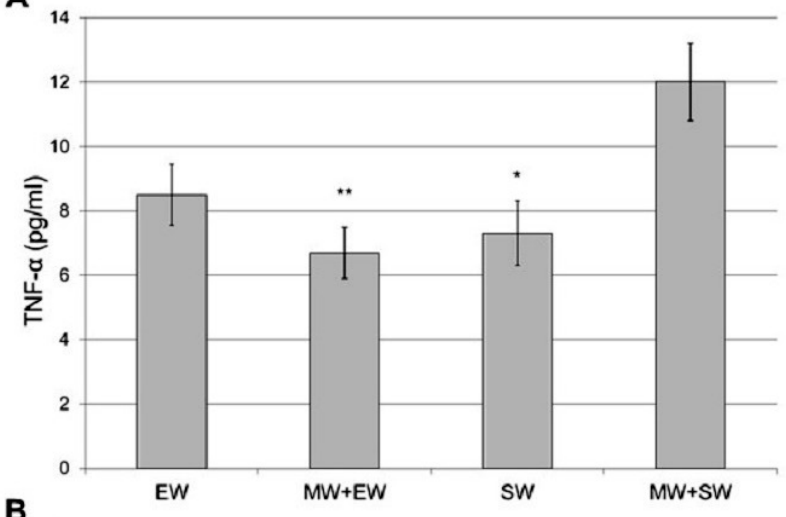

B

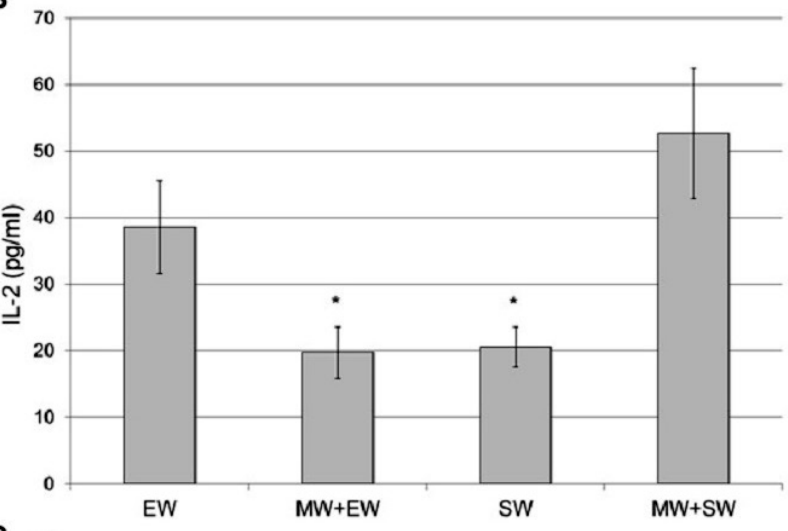

C

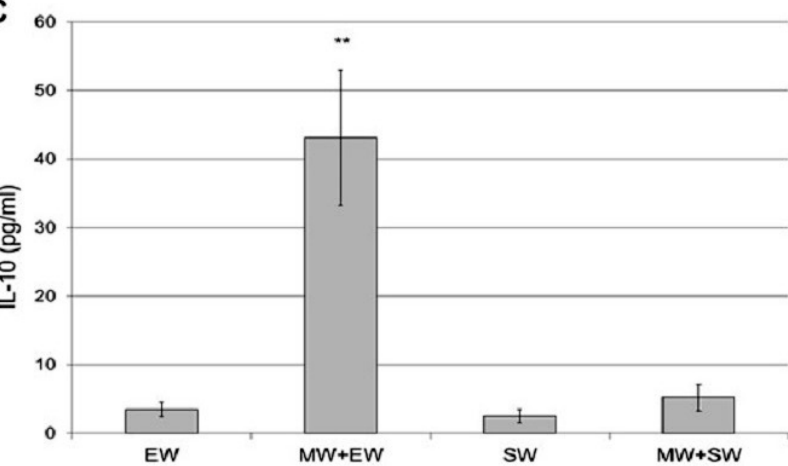

Figure 1. Concentration of TNF- $\alpha$ (A), IL-2 (B) and IL-10 (C) in the serum of the rats at the end of the 90 -day study period. The data are expressed as mean \pm standard error $(n=7) .{ }^{*} p<0.05$, ${ }^{* *} p<$ 0.01 compared to MW+SW, determined by Student's $t$-test. MW, microwave; EW, sham-exposed rats with experimental (silica-rich) water supply; MW+EW, MW-exposed with silica-rich water supply; SW, sham-exposed rats with standard water supply; MW+SW, MW-exposed with standard water supply. serum IL-2 levels (Figure 1B). The MW+EW rats had significantly higher systemic IL-10 levels than EW controls (43.1 $\pm 22 v s .3 .5 \pm 2.4 \mathrm{pg} / \mathrm{ml}), \mathrm{SW}$ and $\mathrm{MW}+\mathrm{SW}$ groups $(p<0.01)$ (Figure 1C). This MW effect was not observed in MW+SW rats when compared to SW controls. The results show that silica-rich water enhanced the chronic MW exposure mediated increase in serum IL-10 levels. The serum sICAM-1 and sVCAM-1 levels were not changed, regardless of MW radiation or the water type used.

\section{The effects of chronic $M W$ exposure and silica intake on PMs function}

The long-term MW exposure significantly increased nonstimulated PMs phagocytic activity that was observed through the increased intake of Natural Red particles. Phagocytic activity of the LPS-stimulated PMs was preserved in $\mathrm{MW}+\mathrm{EW}$, but decreased in $\mathrm{MW}+\mathrm{SW}$ group, suggesting that silica-rich water preserves a physiological level of phagocytic capability of stimulated macrophages in MW exposed rats (Figure 2). The non-stimulated MW exposed PMs produced higher TNF- $\alpha$ levels than their non-irradiated controls (EW and SW). This effect was partially ameliorated by the silicarich water intake (higher TNF- $\alpha$ in $\mathrm{MW}+\mathrm{SW}$ than $\mathrm{MW}+\mathrm{EW}$, $p<0.01)$. The silica-rich water in animals, not exposed to the MW, seem to restrict TNF- $\alpha$ increase caused by LPS (higher TNF- $\alpha$ in SW than EW, $p<0.01$ ), implying that silica-rich water suppresses proinflammatory activation of PMs by LPS. On the other hand, LPS-stimulated MW exposed PMs had lower TNF-a levels than their sham controls (EW and SW). Thus, the MW exposition suppressed LPS potential

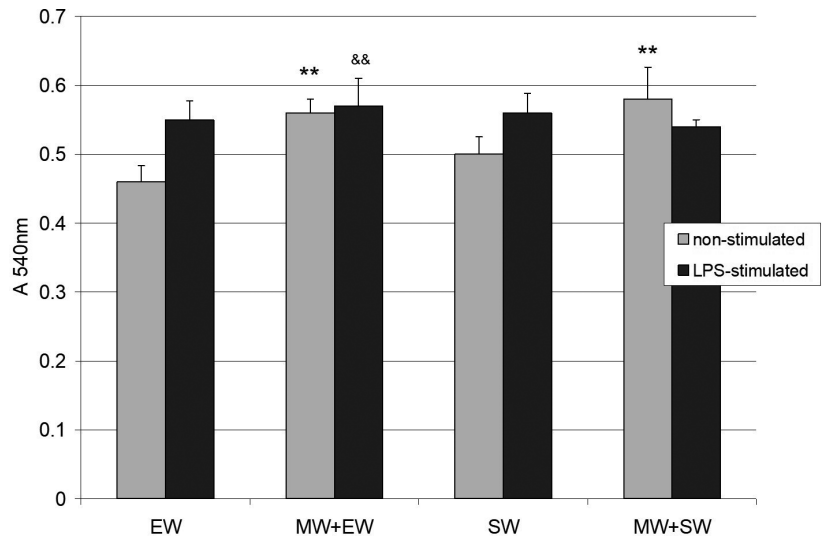

Figure 2. Phagocytic activity of the PMs in the rat groups, as measured by Neutral Red uptake. The data are expressed as mean \pm standard error $(n=7)$. The non-stimulated PMs from both MW exposed groups had higher phagocytic activity than the PMs of their controls ( ${ }^{* *} p<0.01 v$ s. EW and SW, respectively). ${ }^{\& \&} p<0.05$ $v s$. LPS-stimulated MW+SW, as determined by Student's $t$-test. For abbreviations, see Fig. 1. 
for TNF- $\alpha$ synthesis in both water type groups, with greater suppression in animals that took standard water (Figure 3A). The non-stimulated MW exposed PMs produced significantly more IL-10 than sham exposed rats, in both water type groups $(p<0.01)$. Sole LPS stimulation (without MW) induced IL-10 production in both PMs cultures, but significantly less in animals that took EW than SW. The LPS-stimulated PMs from MW+EW rats produced more IL-10 than their EW counterparts, while MW+SW PMs had significantly lower IL-10 production compared to the corresponding SW controls (both $p<0.05$ ). Also, LPS-stimulated $\mathrm{MW}+\mathrm{EW}$ showed higher IL-10 production than MW+SW cells $(p<0.05)$ (Figure 3B).

\section{Discussion}

Important role of the immune system is defense against environmental pollutants. This makes components of the immune system suitable candidates for research on the MW radiation health effects. Current data on the MW exposure report only subtle cellular changes that could not be detected using basic immune functioning tests. Therefore, more sensitive and specific tests need to be identified, such as measurement of the cytokine release (Szmigielski 2013). Several studies of short-term GSM mobile phones MW exposure did not find significant alterations in activity and competence of lymphocytes in vitro (Tuschl et al. 2006) and in vivo (Gatta et al. 2003). In vivo exposure to a short-term GSM modulated $900 \mathrm{MHz}$ radio frequency field in mice showed no effects on $B$ cell peripheral differentiation, antibody production, nor antigen specific IgG, which requires T-B interaction (Nasta et al. 2006). These results imply that primary targets of MW on immune system are not lymphocytes and their subpopulations, but the cells of innate immunity such as residential macrophages and neutrophils that can further influence on adaptive immunity, in a way that short-term MW exposure may activate, while prolonged exposure may inhibit both humoral and cellular immune functions (Grigoriev et al. 2010). Our results show a dual effect of 3 months $900 \mathrm{MHz}$ GSM MW radiation on the serum TNF- $\alpha$ and IL-2 levels, that was also dependent on a silica concentration in drinking water. TNF- $\alpha$ is involved in systemic inflammation and is mainly secreted by activated macrophages (Locksley et al. 2001). In our study, TNF-a levels increased in the MW exposed rats, similarly to the study of Megha et al. (2012) who observed TNF- $\alpha$ increase in 30 days after $900 \mathrm{MHz}$ MW exposure in the hippocampal brain tissue of Fischer rats. However, the TNF- $\alpha$ and IL-2 levels were significantly lower in our MW exposed rats that drank silicon-rich water than those with low silica water supply. This indicates that silicon-rich water suppresses MW-induced systemic increase of proinflammatory cytokines in these animals. Peripheral
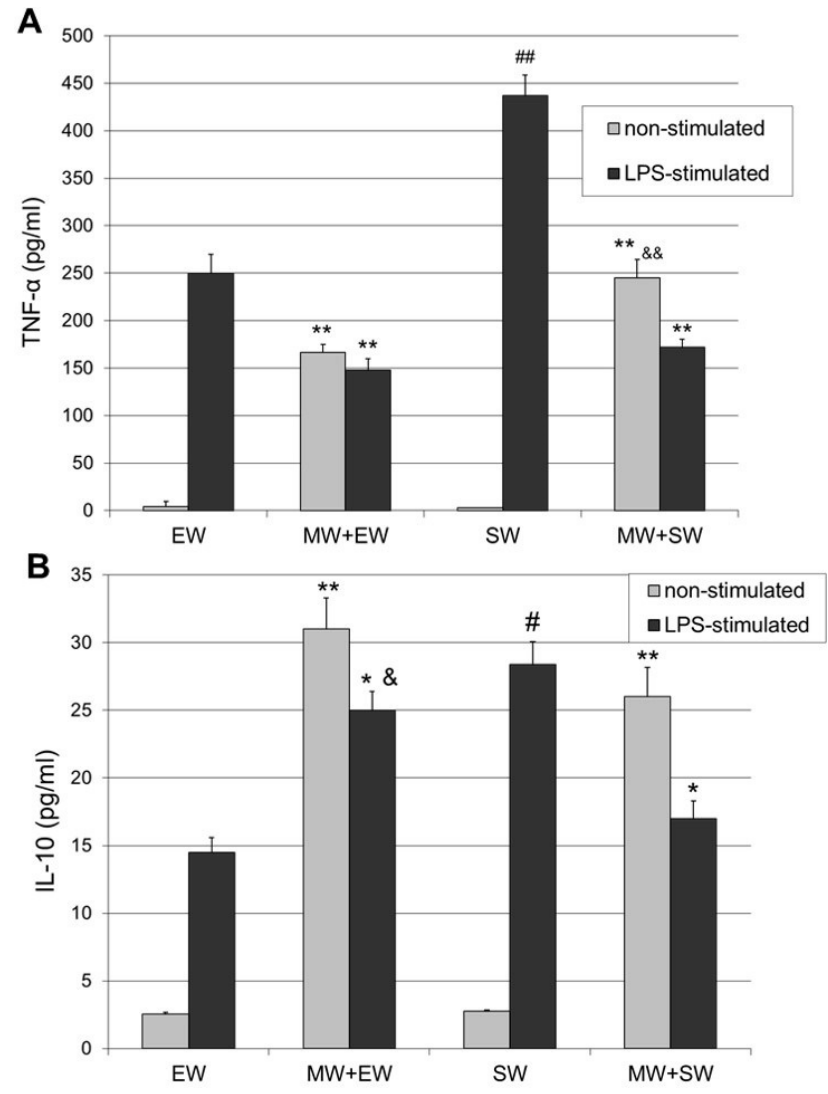

Figure 3. Production of TNF- $\alpha$ (A) and IL-10 (B) by the PMs from the rat groups. The data are expressed as mean \pm standard error $(n=7)$; $\mathrm{P}$ values were determined by Student's $t$-test. ${ }^{*} p<0.05$, ${ }^{* *} p<0.01 \mathrm{MW}+\mathrm{EW}$ and $\mathrm{MW}+\mathrm{SW} v s$. non-stimulated EW and SW, respectively; ${ }^{\&} p<0.05 v s$. LPS-stimulated MW+SW; ${ }^{*} \& p<$ 0.01 vs. non-stimulated MW+EW; ${ }^{\#} p<0.05,{ }^{\# \#} p<0.01$ vs. LPSstimulated EW.

blood mononuclear cells exposed to $900 \mathrm{MHz}$ simulated GSM signal showed significantly higher response to mitogens and had higher immunogenic activity than controls, suggesting intensified immune activity (Stankiewicz et al. 2006). The chronic MW exposure in our study significantly increased phagocytic activity and TNF- $\alpha$ secretion of the non-stimulated PMs. One of the earlier observation showed that MW treatment of NMRI mice $(8.15-18 \mathrm{GHz}$ at a power density of $1 \mathrm{~W} / \mathrm{cm}^{2}$ ) increased TNF production more effectively than the LPS stimulation (Fesenko et al. 1999). Similarly, the summarized results of 10 experiments determined significant increase of monocytes activity under the influence of $900 \mathrm{MHz}$ MW (GSM $20 \mathrm{~V} / \mathrm{m}$, SAR $0.024 \mathrm{~W} / \mathrm{kg}$, for 15 min daily) (Stankiewicz et al. 2009). In our study, the silica-rich water supply protected PMs' phagocytic capability and decreased their TNF- $\alpha$ secretion after LPS application or MW exposition. In contrast, amorphous silica particles 
induce strong IL- $1 \beta$ response from mouse macrophages in the presence of LPS (Sandberg et al. 2012), ortho-silicic acid showed immunostimulating effects (Koo et al. 2006), while 6-pentadecylsalicylic acid increased mouse PMs activity and TNF- $\alpha$ and IL- 6 secretion, and decreased IL- 4 and IL-10 (Gnanaprakasam et al. 2015). After LPS stimulation, in our experiment, MW exposed PMs secreted lower amounts of TNF- $\alpha$ than non-irradiated sham controls, but higher levels were produced by PMs from animals with silica-rich than standard water. The results demonstrate the silica-rich water inhibiting effect toward proinflammatory action of both MW and LPS. However, as the MW exposition suppressed LPS activating potential on PMs, the better response of EW irradiated cells to LPS might be also the result of silica-rich water protective effect against MW. Chronic MW is described to act directly immuno-stimulatory on PMs or through the alteration of PMs subpopulations. Two sets of PMs with distinct properties exist in mice (Ghosn et al. 2010). Small PMs exert proinflammatory characteristics and are major source of the inflammatory mediators in response to LPS, whereas large PMs appear to have a role in the maintenance of peritoneal physiological conditions with greater phagocytic activity for apoptotic cells (Cain et al. 2013; Cassado et al. 2015). LPS is a relevant factor that polarizes macrophage population toward proinflammatory phenotype and may augment acute inflammatory response to the silica particles (Sandberg et al. 2012). However, $\mathrm{SiO}_{2}$ forms in silica-rich water could preserve large PMs predominance and hence decrease TNF- $\alpha$ synthesis after MW, but sustain phagocytic reactivity of LPS-stimulated PMs, which is observed in our experiment. IL-10 is an anti-inflammatory cytokine mainly produced by monocytes. It counteracts inflammation by inhibiting the synthesis of pro-inflammatory Th1 cytokines. According to our results, the long-term MW exposure significantly raised serum IL-10 in rats with the silica-rich water intake and markedly induced its production in both non-stimulated PMs water groups. The LPS stimulation, in the absence of MW irradiation, significantly raised PMs' IL-10 production in the rats with SW compared to EW intake, pointing to the restricting effect of silica-rich water toward LPS-stimulation. However, MW irradiated PMs produced more IL-10 after the stimulation in EW groups in the contrary to the SW groups. It seems that silica-rich water tends to keep the relation of the cytokine production as previous. Similarly, to the observations with TNF, there is a possibility that silica-rich water ameliorates MW suppressive effect towards LPS reactivity. Current explanations regarding cytokine alterations under the MW are various, often contradictory, and depending on a duration and intensity of the MW radiation. Dabrowski et al. (2003) reported increased IL-1 $\beta$ and IL-10 production in the microculture system of human blood mononuclear cells pre-exposed to $1300 \mathrm{MHz}$ pulse-modulated MWs, indicating an immunotropic influence and proinflammatory activity even at the low levels of exposure. On the contrary, locally (ipsilateral) decreased IL-10 concentrations were reported in the saliva from subjects who used cell phones for more than 10 years. These alterations were suggested to be due to the MWs' heating effects that may change cytokines expression levels (Siqueira et al. 2016). There are limited data on the effects of chronic mobile phone MW exposure on cell adhesion molecules and vascular biology. The short-term (20 min) pulse modulated $900 \mathrm{MHz}$ and $1800 \mathrm{MHz}$ radiofrequency radiation were shown to increase the permeability of blood brain barrier in rats (Sirava et al. 2016), which could alter endothelium-microglial interaction with inflammation related processes including increased expression of adhesive molecules (Da Fonseca et al. 2014). However, our experiment did not register disturbances in serum soluble ICAM-1 and VCAM-1 levels, nor the influence of silica content on their concentration.

Radio frequency/MW radiations are proposed to be possible carcinogen for humans (Baan et al. 2011). Accordingly, the mice chronically exposed to mobile phone MW radiation showed increased incidence of tumors (Lerchl et al. 2015). Because carcinogenesis is partly explained through the increased TNF- $\alpha$ and IL- 6 production by residential and PMs (Nakagawa et al. 2014), it would be interesting to address the question of modifying silica-rich water effects on inflammation and carcinogenesis. In conclusion, this is the first study to show functional and secretory activity of PMs influenced by the silica-rich water in rats chronically exposed to the $900 \mathrm{MHz} \mathrm{MW}$ radiation. Our results show, that silica-rich water preserves the TNF- $\alpha$ and IL-2 systemic levels which would be increased under the long-term MW exposure. At the same time, silica-rich water supply enhanced the chronic MW exposure mediated increase in serum IL-10 levels. It also preserved a physiological level of macrophages' phagocytic capability, mediated reversal of the MW induced TNF- $\alpha$ production and kept the relation of IL-10 production in MW exposed stimulated PMs as previous. The silica-rich water opposed pro-inflammatory cytokines production and it could be a relevant modulator of chronic MW radiation effects on systemic and peritoneal inflammation.

Acknowledgements. The study is supported under the Projects no. 43012 and 41018 of Ministry of Education, Science and Technological Development of the Republic of Serbia.

Conflict of interest: None.

\section{References}

Baan R, Grosse Y, Lauby-Secretan B, El Ghissassi F, Bouvard V, Benbrahim-Tallaa L, Guha N, Islami F, Galichet L, Straif K (2011): WHO International Agency for Research on Cancer 
Monograph Working Group: Carcinogenicity of radiofrequency electromagnetic fields. Lancet. Oncol. 12, 624-626 https://doi.org/10.1016/S1470-2045(11)70147-4

Cain DW, O'Koren EG, Kan MJ, Womble M, Sempowski GD, Hopper K, Gunn MD, Kelsoe G (2013): Identification of a tissue-specific, C/EBPbeta-dependent pathway of differentiation for murine peritoneal macrophages. J. Immunol. 191, 4665-4675 https://doi.org/10.4049/jimmunol.1300581

Cassado AA, D'Império Lima MR, Bortoluci KR (2015): Revisiting mouse peritoneal macrophages: heterogeneity, development, and function. Front. Immunol. 6, 225 https://doi.org/10.3389/fimmu.2015.00225

Da Fonseca ACC, Matias D, Garcia C, Amaral R, Geraldo LH, Freitas C, Lima FR (2014): The impact of microglial activation on blood-brain barrier in brain diseases. Front. Cell. Neurosci. 8, 362 https://doi.org/10.3389/fncel.2014.00362

Dabrowski MP, Stankiewicz W, Kubacki R, Sobiczewska E, Szmigielski S (2003): Immunotropic effects in cultured human blood mononuclear cells preexposed to low-level 1300 $\mathrm{MHz}$ pulse-modulated microwave field. Electromagn. Biol. Med. 22, 1-13 https://doi.org/10.1081/JBC-120020347

Fesenko EE, Makar VR, Novoselova EG, Sadovnikovb VB (1999): Microwaves and cellular immunity. I. Effect of whole body microwave irradiation on tumor necrosis factor production in mouse cells. Bioelectrochem. Bioenerg. 49, 29-35 https://doi.org/10.1016/S0302-4598(99)00058-6

Gatta L, Pinto R, Ubaldi V, Pace L, Galloni P, Lovisolo GA, Marino C, Pioli C (2003): Effects of in vivo exposure to GSM-modulated $900 \mathrm{MHz}$ radiation on mouse peripheral lymphocytes. Radiat. Res. 160, 600-605 https://doi.org/10.1667/RR3078

Ghosn EE, Cassado AA, Govoni GR, Fukuhara T, Yang Y, Monack DM, Bortoluci KR, Almeida SR, Herzenberg LA, Herzenberg LA (2010): Two physically, functionally, and developmentally distinct peritoneal macrophage subsets. Proc. Natl. Acad. Sci. USA $107,2568-2573$ https://doi.org/10.1073/pnas.0915000107

Gnanaprakasam JN, Estrada-Mu-iz E, Vega L (2015): The anacardic 6-pentadecyl salicylic acid induces macrophage activation via the phosphorylation of ERK1/2, JNK, P38 kinases and NF- $\kappa B$. Int. Immunopharmacol. 29, 808-817 https://doi.org/10.1016/j.intimp.2015.08.038

Grigoriev YG Grigoriev OA, Ivanov AA, Lyaginskaya AM, Merkulov AV, Shagina NB, Maltsev VN, Lévêque P, Ulanova AM, Osipov VA, Shafirkin AV (2010): Confirmation studies of Soviet research on immunological effects of microwaves: Russian immunology results. Bioelectromagnetics 31, 589-602 https://doi.org/10.1002/bem.20605

Koo H, Ryu SH, Ahn HJ, Jung WK, Park YK, Kwon NH, Kim SH, Kim JM, Yoo BW, Choi SI, Davis WC, Park YH (2006): Immunostimulatory effects of the anionic alkali mineral complex BARODON on equine lymphocytes. Clin. Vaccine. Immunol. 13, 1255-1266 https://doi.org/10.1128/CVI.00150-06
Lerchl A, Klose M, Grote K3 Wilhelm AF, Spathmann O, Fiedler T, Streckert J, Hansen V, Clemens M (2015): Tumor promotion by exposure to radiofrequency electromagnetic fields below exposure limits for humans. Biochem. Biophys. Res. Commun. 459, 585-590 https://doi.org/10.1016/j.bbrc.2015.02.151

Locksley RM, Killeen N, Lenardo MJ (2001): The TNF and TNF receptor superfamilies: integrating mammalian biology. Cell 104, 487-501 https://doi.org/10.1016/S0092-8674(01)00237-9

Megha K, Deshmukh PS, Banerjee BD, Tripathi AK, Abegaonkar MP (2012): Microwave radiation induced oxidative stress, cognitive impairment and inflammation in brain of Fischer rats. Indian. J. Exp. Biol. 50, 889-896

Munjas Jurkić L, Cepanec I, Kraljević Pavelić S, Pavelić K (2013): Biological and therapeutic effects of ortho-silicic acid and some ortho-silicic acid-releasing compounds: New perspectives for therapy. Nutr. Metab. (Lond) 10, 2 https://doi.org/10.1186/1743-7075-10-2

Nakagawa H, Umemura A, Taniguchi K, Font-Burgada J, Dhar D, Ogata H, Zhong Z, Valasek MA, Seki E, Hidalgo J, et al. (2014): ER stress cooperates with hypernutrition to trigger TNF-dependent spontaneous HCC development. Cancer Cell 26, 331-343 https://doi.org/10.1016/j.ccr.2014.07.001

Nasta F, Prisco MG, Pinto R, Lovisolo GA, Marino C, Pioli C (2006): Effects of GSM-modulated radiofrequency electromagnetic fields on B-cell peripheral differentiation and antibody production. Radiat. Res. 165, 664-670 https://doi.org/10.1667/RR3555.1

Repetto G, del Peso A, Zurita JL (2008): Neutral red uptake assay for the estimation of cell viability/cytotoxicity. Nat. Protoc. 3, $1125-1131$ https://doi.org/10.1038/nprot.2008.75

Robberecht H, Van Cauwenbergh R, Van Vlaslaer V, Hermans N (2009): Dietary silicon intake in Belgium: Sources, availability from foods, and human serum levels. Sci. Total. Environ. 407, 4777-4782 https://doi.org/10.1016/j.scitotenv.2009.05.019

Sandberg WJ, Lag M, Holme JA, Friede B, Gualtieri M, Kruszewski M, Schwarze PE, Skuland T, Refsnes M (2012): Comparison of non-crystalline silica nanoparticles in IL- $1 \beta$ release from macrophages. Part. Fibre. Toxicol. 9, 32

https://doi.org/10.1186/1743-8977-9-32

Siqueira EC, de Souza FT, Ferreira E, Souza RP, Macedo SC, Friedman E, Gomez MV, Gomes CC, Gomez RS (2016): Cell phone use is associated with an inflammatory cytokine profile of parotid gland saliva. J. Oral. Pathol. Med. 45, 682-686 https://doi.org/10.1111/jop.12434

Sirava B, Seyhana N (2016): Effects of GSM modulated radiofrequency electromagnetic radiation on permeability of blood-brain barrier in male \& female rats. J. Chem. Neuroanat. $75,123-127$ https://doi.org/10.1016/j.jchemneu.2015.12.010

Stankiewicz W, Dabrowski MP, Kubacki R, Sobiczewska E, Szmigielski S (2006): Immunotropic influence of $900 \mathrm{MHz} \mathrm{MW}$ GSM signal on human blood immune cells activated in vitro. Electromagn. Biol. Med. 25, 45-51 
https://doi.org/10.1080/15368370600572961

Stankiewicz W, Dąbrowski MP, Kieliszek J, Sobiech J (2009): Low energy electromagnetic fields and immunity. Innt. Rev. Allergol. Clin. Immunol. 15, 36-41

Szmigielski S (2013): Reaction of the immune system to lowlevel RF/MW exposures. Sci. Total. Environ. 454-455, 393-400

https://doi.org/10.1016/j.scitotenv.2013.03.034
Tuschl H, Novak W, Molla-Djafari H (2006): In vitro effects of GSM modulated radiofrequency fields on human immune cells. Bioelectromagnetics 27, 188-196 https://doi.org/10.1002/bem.20191

Received: April 22, 2018

Final version accepted: August 27, 2018

First published online: January 18, 2019 